\title{
Impact of neuropathy on the adherence to diabetes-related self-care activities: a cross- sectional study
}

This article was published in the following Dove Press journal:

Patient Preference and Adherence

I July 2016

Number of times this article has been viewed

\author{
Bogdan Timar' \\ Romulus Timar ${ }^{2}$ \\ Adalbert Schiller ${ }^{2}$ \\ Cristian Oancea ${ }^{3}$ \\ Deiana Roman' \\ Mihaela Vlad ${ }^{2}$ \\ Bogdan Balinisteanu ${ }^{4}$ \\ Octavian Mazilu ${ }^{5}$ \\ 'Department of Functional Sciences, \\ ${ }^{2}$ Second Department of Internal \\ Medicine, ${ }^{3}$ Department of Infectious \\ Diseases, ${ }^{4}$ Department of Microscopic \\ Morphology, ${ }^{5}$ First Department of \\ Surgery, "Victor Babes" University of \\ Medicine and Pharmacy, Timisoara, \\ Romania
}

Purpose: The purpose of this study was to evaluate the impact of the presence and severity of neuropathy and depression on the patient's adherence to diabetes-related self-care activities (DRSCA) in a cohort of patients with type 2 diabetes mellitus (T2DM).

Patients and methods: In this cross-sectional, noninterventional study, 198 patients with T2DM were enrolled according to a population-based, consecutive-case enrollment principle. In all patients, the adherence to DRSCA was evaluated using the Summary of Diabetes SelfCare Activities (SDSCA) questionnaire; a higher SDSCA score is associated with a better adherence. The presence and severity of neuropathy was assessed using the Michigan Neuropathy Screening Instrument (MNSI) and the severity of depression using the Patient Health Questionnaire-9 (PHQ-9).

Results: The presence of neuropathy was associated with a decreased SDSCA score (26 points vs 37 points; $P<0.001)$, an increased severe depression prevalence $(24.7 \%$ vs $4.3 \% ; P<0.001)$, and an increased PHQ-9 score (12 points vs 7 points; $P<0.001)$. The MNSI score was reverse correlated with SDSCA score $(r=-0.527 ; P<0.001)$ and positively correlated with PHQ-9 score $(r=0.495 ; P<0.001)$. The reverse correlation between MNSI score and SDSCA score was present for all the subcomponents of SDSCA questionnaire (diet, exercise, glycemic monitoring, and foot care).

Conclusion: The presence of neuropathy is associated with decreases in the quality of adherence to DRSCA in patients with T2DM and with increases in the symptomatology of depression. The significant, negative association between the severity of T2DM and the quality of disease self-management points to a possible loop-type relationship between these two components, being possible a reciprocal augmentation with negative consequences on the global management of the disease.

Keywords: type 2 diabetes mellitus, diabetes self-care, diabetic neuropathy, depression

\section{Introduction}

Currently, diabetes mellitus (DM) is a global public health issue of paramount importance, being estimated that the prevalence of DM is about $10 \%$ worldwide. Nowadays, it is estimated that 415 million people have diabetes, the prediction pointing to $\sim 642$ million by $2040 .^{1,2}$

Diabetes is a leading cause of new blindness cases among adults, kidney failure, and lower limb amputations, excepting those caused by trauma. ${ }^{3}$ Owing to the presence of peripheral neuropathy, patients are often at risk of its complications: falling, presenting ulcerations, or having to amputate a limb. ${ }^{4}$ Sensorimotor distal symmetric polyneuropathy is the most common neurological complication of diabetes, and it is
Second Department of Internal Medicine, "Victor Babes" University of Medicine and Pharmacy, 2 Eftimie Murgu, 30004I Timisoara, Romania Tel +40 74l528093 Fax +40 256462856

Email timar.romulus@umft.ro 
cited to significantly decrease the quality of life in the affected patients. The major risk factors of distal symmetric polyneuropathy are cited to be diabetes duration, hyperglycemia, and age, followed by hypertension, dyslipidemia, and obesity. Height, smoking, insulin resistance, hypoinsulinemia, and others also represent additional risk factors. ${ }^{5}$

Peripheral neuropathy is a long-term complication that causes sensory and motor impairment, negatively affecting the patient's balance, physical functioning, and postural control. Daily activities are influenced by these afflictions, the risk of falling is increased, and by these means, we can observe an increase in morbidity and a decrease in the quality of life. For the best outcomes of rehabilitation programs and prevention of dependence, the early detection of these functional limitations is extremely important. ${ }^{6} \mathrm{~A}$ frequent chronic complication of diabetes is represented by cardiac autonomic neuropathy, ${ }^{7}$ which can have deadly outcomes through the impairment of autonomic nerve fibers responsible for heart rate, myocardial contractility, cardiac electrophysiology, blood vessel increase, or decrease in contractility. Arrhythmias, asymptomatic myocardial ischemia and infarction, intraoperative cardiovascular instability, and increased postinfarction mortality rate are reminded among the wide range of cardiac disorders present in cardiac autonomic neuropathy. The associated clinical symptoms of this complication generally have a late onset, and they include orthostatic hypotension, dizziness, early fatigue and exhaustion during exercise, presyncope, and syncope. ${ }^{8}$ A significant number of studies confirm the correlation between both diabetic neuropathy (DN) and depression and that between the severity of neuropathy and the level of depression, the latter being observed to appear shortly after the debut of diabetic complications, especially peripheral neuropathy. Negative diabetes perceptions, neuropathic pain, and lower perceived personal control have been linked to poor emotional health, increased anxiety, and deterioration of general health status in the diabetic patient. ${ }^{9,10}$

A decrease in glucose self-monitoring and adherence to diet and exercise, as well as an increase in the probability of missing medication doses, was observed in patients with probable major depression compared with other patients. Lack of adherence to diet, exercise, and medication administration was better predicted by severity scores of continuous depressive symptoms compared to probable major depression, which was a better predictor of glucose monitoring. ${ }^{11}$ Having these premises, the aim of our study was to evaluate the impact of the presence and severity of DN on the quality of the adherence to diabetes-related self-care activities
(DRSCA). We hypothesized that a loop-type relationship between these two components may exist: a decreased adherence to DRSCA may alter the glycemic control, thus deteriorating the glycemic control, which will further increase the severity of DN and depression and finally will decrease the adherence to DRSCA even more.

\section{Patients and methods Study design and methods}

In this cross-sectional, non-interventional study, we enrolled 198 patients previously diagnosed with type 2 diabetes mellitus (T2DM), attending scheduled visits in the Center for Diabetes Treatment of the Emergency Hospital Timisoara, Romania. The patients participating in the study were enrolled according to a population-based, consecutive-case principle. The study design, protocol, and informed consent form were reviewed and approved by the Ethics Committee of the Emergency Hospital Timisoara", approval number 328/2014; all patients provided written informed consent prior to any study procedure or activity.

At the time of screening the patients, the following were considered as the exclusion criteria: the inability to provide informed consent, inability to provide accurate anamnestic medical history data, prior history of nondiabetic neuropathies, major cardiovascular events (according to Hicks 2014 criteria) 3 months prior to screening, or any other condition which, in the investigators' opinion, could lead to biases in the study results.

The enrolled patients had a median age of 61 years, a median duration of diabetes of 7 years, and $44.9 \%$ of them $(n=89)$ were males. Since the studied sample has similar characteristics when compared to the Romanian T2DM patient population and since the enrollment criterion in the study was a population-based consecutive-case principle, we consider that the results of this study are representative of the entire Romanian population of patients with T2DM.

\section{DN assessment}

The Michigan Neuropathy Screening Instrument (MNSI) was used to diagnose DN. MNSI is a validated score instrument for $\mathrm{DN}$, being widely used for the diagnosis and quantification of distal symmetrical peripheral neuropathy in diabetes. The MNSI includes two separate assessments: a 15-item self-administered questionnaire that is scored with regard to abnormal responses provided by the patient and a lower extremity examination that consists of inspection and assessment of vibratory sensation and ankle reflexes. The MNSI provides better sensitivity and specificity than the individual $\mathrm{DN}$ tests separately; the diagnosis of DN was considered 
positive in the case of a questionnaire score $\geq 7$ or in the case a clinical examination score $\geq 2.5$. A higher MNSI score is associated with a more severe DN. ${ }^{12}$

\section{Diabetes-related self-care activities}

The quality of DRSCA was evaluated using the Summary of Diabetes Self-Care Activities Questionnaire (SDSCA), which is a validated tool in the population of DM patients. A higher SDSCA score is associated with better disease self-care in these patients. The SDSCA questionnaire includes items assessing the following aspects of the diabetes care: general and specific diet measures, exercise, blood-glucose testing, foot care, and smoking. It was proven that the SDSCA questionnaire is a brief yet reliable and valid self-report measure of diabetes self-management, useful for both research and clinical practice. ${ }^{13}$

\section{Clinical, anthropometric, and laboratory data}

Data regarding patient age; diabetes history, including the history of diabetes treatment; smoking history; and body mass index were collected from the patients' medical records. The hemoglobin A1c (HbA1c) level was measured using a National Glycohemoglobin Standardization Program (NGSP)-standardized and Diabetes Control and Complications Trial (DCCT)-compliant immune turbidimetric assay (Hoffman-La Roche Ltd., Basel, Switzerland), having an intermeasurement coefficient of variation of $1.64 \%$ according to the manufacturer's specifications. The presence and severity of chronic kidney disease was diagnosed according to the Kidney Disease: Improving Global Outcomes 2012 guidelines. Retinopathy diagnosis was established after performing a funduscopic examination by the same trained ophthalmologist specialized in the diagnosis and treatment of diabetic eye complications.

To evaluate the presence and the severity of depression, we used the Patient Health Questionnaire-9 (PHQ-9). With its nine items, the PHQ-9 test consists of the actual nine criteria on which the diagnosis of Diagnostic and Statistical Manual of Mental Disorders IV (DSM-IV) depressive disorders is based. The PHQ-9 is a dual-purpose instrument that can establish, with the same nine items, depressive distress as well as grade of depressive symptom severity. A higher PHQ-9 score is associated with more severe depression; based on this score, the depression's severity may be divided into three groups: minimal or mild (PHQ-9 score $<10$ ), moderate (PHQ-9 score 10-19), and severe (PHQ-9 score $>19) .{ }^{14}$

\section{Statistical analysis}

Data were collected and analyzed using SPSS v17 statistical software package (SPSS Inc., Chicago, IL, USA) and were presented as average \pm standard deviation (numerical variables with Gaussian distribution), median (interquartile range; numerical variables with nonparametric distributions), and number of individuals (percentage from the subgroup total). To assess the significance of the differences between groups, the Student's $t$-test (mean, Gaussian populations), Mann-Whitney $U$-test (median, nonparametric populations), and chi-square test (proportions) were used. Distributions of continuous variables were tested for normality using the Shapiro-Wilk test and for equality of variances using the Levene's test.

To evaluate the strength of the association between two continuous variables, we used Spearman's correlation coefficient; the statistical significance of the correlation was evaluated using $t$-distribution test.

A $P$-value $<0.05$ was considered the threshold of statistical significance.

\section{Results}

In our study cohort, the prevalence of $\mathrm{DN}$, as diagnosed according to the MNSI criteria, was $28.8 \%$ (57 cases). The presence of overt neuropathy was associated with an increased age (64.5 years vs 59 years; $P=0.001)$, BMI (31.9 kg/m ${ }^{2}$ vs $\left.29.9 \mathrm{~kg} / \mathrm{m}^{2} ; P=0.030\right)$, and depression severity - assessed using the PHQ-9 score (12.2 points vs 7.2 points; $P=0.011)$. Patients with a positive diagnosis of DN had a higher prevalence of other diabetes complications: chronic kidney disease $(56.1 \%$ vs $14.2 \% ; P<0.001)$ and retinopathy $(54.4 \%$ vs $22.0 \%)$. The other studied parameters had no significant differences between the groups: diabetes duration, $\mathrm{HbA1c}$, prevalence of smoking, and hypertension (Table 1).

In our study, the presence of neuropathy was associated with significant decreases in the quality of diabetes selfmanagement (SDSCA global score 26 points vs 37 points in patients without neuropathy, $P<0.001$; Figure 1). The decreases in diabetes self-management were significant for all the studied subitems: diet-related self-management (12 points vs 17 points; $P<0.001$ ), physical exercise ( 5 points vs 6 points; $P=0.002$ ), glycemic values monitoring ( 4 points vs 8 points; $P=0.010$ ), and foot care ( 3 points vs 6 points; $P=0.003$ ).

The group of patients with neuropathy had a significantly higher median of PHQ-9 depression score (12 points vs 7 points; $P<0.001$; Figure 2 ). 
Table I Patient's characteristics, stratified according to the presence of DN

\begin{tabular}{|c|c|c|c|}
\hline & Without overt neuropathy & Overt neuropathy present & $P$-value \\
\hline Male sex ${ }^{a}$ & $64(45.4 \%)$ & $25(43.9 \%)$ & 0.845 \\
\hline Age (years) ${ }^{b}$ & $59(12)$ & $64.5(10.5)$ & $0.001 *$ \\
\hline Diabetes duration (years) ${ }^{b}$ & $7(9)$ & $7(9)$ & 0.867 \\
\hline $\mathrm{HbAlc}(\%)^{c}$ & $8.0 \pm 1.8$ & $8.6 \pm 1.6$ & 0.620 \\
\hline BMI $\left(\mathrm{kg} / \mathrm{m}^{2}\right)^{\mathrm{c}}$ & $29.9 \pm 4.3$ & $31.9 \pm 3.9$ & $0.030 *$ \\
\hline PHQ-9 score (points) ${ }^{c}$ & $7.2 \pm 5.3$ & $12.2 \pm 7.9$ & $0.011 *$ \\
\hline Smokers ${ }^{\mathrm{a}}$ & $50(35.5 \%)$ & $15(26.3 \%)$ & 0.215 \\
\hline Hypertensive patients $^{\mathrm{a}}$ & II (83.0\%) & 45 (78.9\%) & 0.505 \\
\hline Chronic kidney disease $^{a}$ & $20(14.2 \%)$ & $32(56.1 \%)$ & $<0.001 *$ \\
\hline Retinopathy ${ }^{\mathrm{a}}$ & $31(22.0 \%)$ & $31(54.4 \%)$ & $<0.00$ I* \\
\hline
\end{tabular}

Notes: *Differences are statistically significant at $\alpha<0.05$ threshold. aDichotomous variables. Results are presented as number of individuals (percentage from the subgroup). $P$-value was calculated using the chi-square test. ${ }^{b} \mathrm{Numerical}$ variables with nonparametric distribution. Results are presented as median (interquartile range). $P$-value was calculated using the Mann-Whitney U-test. cNumerical variables with Gaussian distribution. Results are presented as average \pm standard deviation. $P$-value was calculated using the unpaired Student's t-test.

Abbreviations: DN, diabetic neuropathy; HbAlc, hemoglobin Alc; BMI, body mass index; PHQ-9, Patient Health Questionnaire-9.

Furthermore, we noted a different trend in the prevalence of depression's severity: most patients without neuropathy had no or only mild depression ( $32.6 \%$ vs $44.7 \%$ ) and only $4.3 \%$ of them had severe depression compared with the prevalence of severe depression, of $24.6 \%$, in the group of patients with neuropathy (Table 2$)$. The differences in trends were statistically significant $(P<0.001)$.

The diabetes self-care score was reversely and significantly correlated with the severity of neuropathy, assessed using the MNSI score (Spearman's $r=-0.527 ; P<0.001$; Figure 3), which revealed the fact that patients with more severe neuropathy tend to have less diabetes-related selfmanagement activities.

The SDSCA scores, including all studied subcomponents, reverse correlated with global, questionnaire, and clinical assessment MNSI scores, revealing a negative

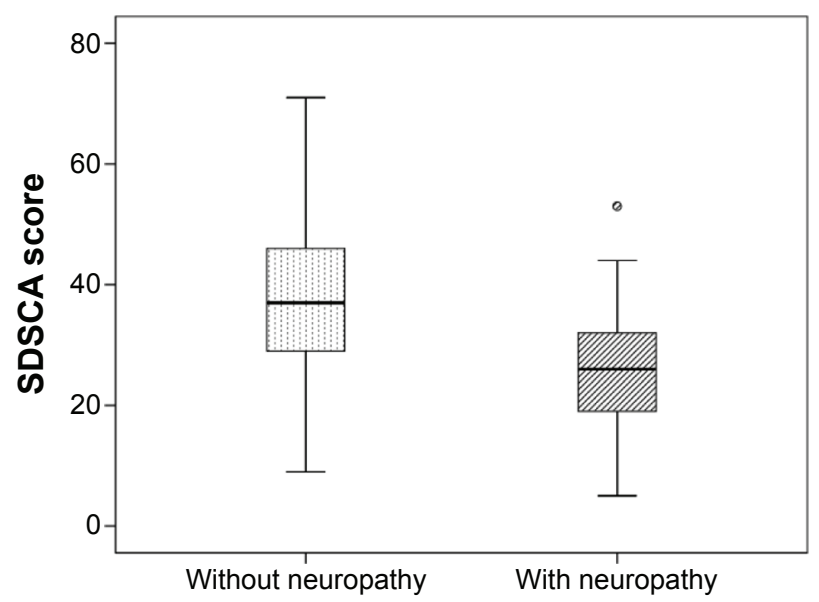

Figure I SDSCA adherence in patients with vs without DN.

Abbreviations: SDSCA, Summary of Diabetes Self-Care Activities; DN, diabetic neuropathy. association between the severity of neuropathy and the quality of diabetes-related self-management activities. All correlations, excepting the one between SDSCA foot care and MNSI questionnaire, were statistically significant (Table 3). With the exception of foot care self-management, the global MNSI score correlated more strongly with all diabetes self-management items compared to MNSI questionnaire or MNSI clinical evaluation component, this being most probably a consequence of the improvement obtained in diagnosis and evaluation of neuropathy by using the two methods combined rather than each of them used alone.

\section{Discussion}

This study showed that T2DM patients with neuropathy had lower self-care scores, being less inclined to maintain a proper diet, exercise routine, and glycemic monitoring. This tendency was correlated with the severity of the neuropathy.

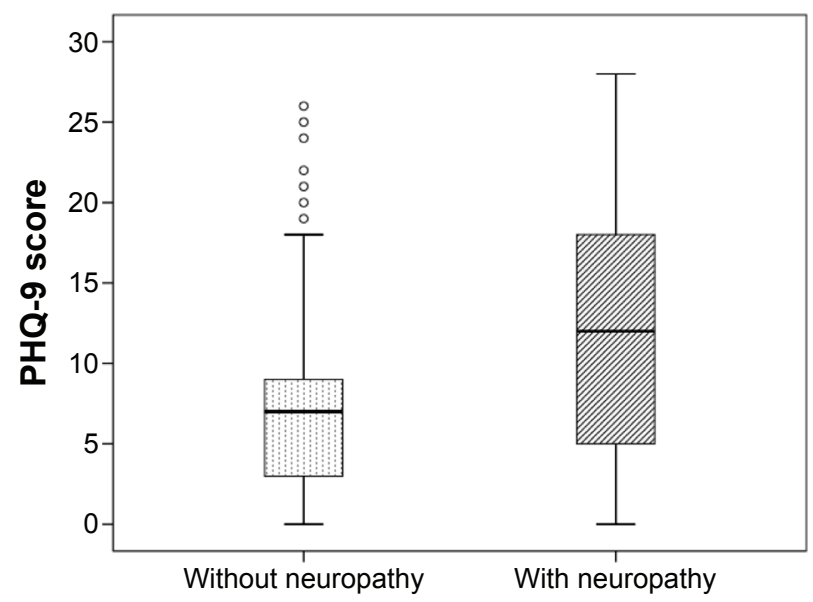

Figure 2 Depression severity in patients with vs without DN. Abbreviations: DN, diabetic neuropathy; PHQ-9, Patient Health Questionnaire-9. 
Table 2 SDSCA adherence and depression's severity in patients with vs without DN

\begin{tabular}{|c|c|c|c|}
\hline & Neuropathy absent $(n=\mid 4 I)$ & Neuropathy present $(n=57)$ & $P$-value \\
\hline SDSCA global score & $37(17)$ & $26(14)$ & $<0.00 I^{*}$ \\
\hline SDSCA diet score ${ }^{\mathrm{a}}$ & $17(8)$ & $12(8)$ & $<0.00 I^{*}$ \\
\hline SDSCA exercise score ${ }^{a}$ & $6(5)$ & $5(3)$ & $0.002 *$ \\
\hline SDSCA glycemic monitoring score ${ }^{a}$ & $8(12)$ & $4(6)$ & $0.010 *$ \\
\hline SDSCA foot care score ${ }^{a}$ & $6(8)$ & $3(4)$ & $0.003 *$ \\
\hline PHQ-9 score ${ }^{a}$ & $7(6)$ & $12(14)$ & $<0.00 I^{*}$ \\
\hline Minimal or no depression ${ }^{b}$ & $32.6 \%(46)$ & $22.8 \%(13)$ & $<0.00 I^{*}$ \\
\hline Mild depression ${ }^{\mathrm{b}}$ & $44.7 \%(63)$ & $17.5 \%(10)$ & \\
\hline Moderate depression ${ }^{\mathrm{b}}$ & $12.8 \%(18)$ & $22.8 \%(13)$ & \\
\hline Moderately severe depression ${ }^{\mathrm{b}}$ & $5.7 \%(8)$ & $12.3 \%(7)$ & \\
\hline Severe depression ${ }^{\mathrm{b}}$ & $4.3 \%(6)$ & $24.6 \%(14)$ & \\
\hline
\end{tabular}

Notes: *Differences are statistically significant at $\alpha<0.05$ threshold. ${ }^{2}$ Numerical variables with nonparametric distribution. Results are presented as median (interquartile range). $P$-value was calculated using the Mann-Whitney $U$-test. 'bichotomous variables. Results are presented as number of individuals (percentage from the subgroup). $P$-value was calculated using the chi-square test.

Abbreviations: DN, diabetic neuropathy; SDSCA, Summary of Diabetes Self-Care Activities; PHQ-9, Patient Health Questionnaire-9.

Moreover, the severity of the neuropathy is directly linked to the degree of depression: a smaller percentage of patients presenting neuropathy experience no or mild depression compared to those without neuropathy and a larger percentage present moderate, moderately severe, or severe depression compared to those who do not have neuropathy.

Among the predictors of the late-onset complications of diabetes, hyperglycemia has been shown to be a major one, its control having been proven of crucial importance in the prevention of vascular events. In spite of blood-glucose levels fluctuating with a series of internal and external factors, achieving an ideal outcome regarding blood glucose is ensured by the patient's active disease management, it being widely accepted that adequate self-management prevents the complications of both type 1 diabetes mellitus and T2DM. ${ }^{15}$ Exhibiting a decrease in self-care scores, patients do not achieve the possible statistically significant and clinically

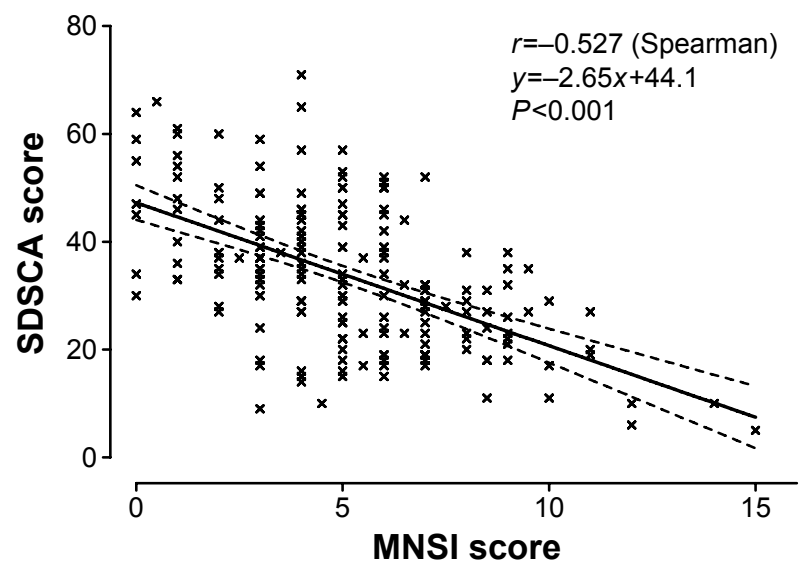

Figure 3 Correlation between the severity of DN and SDSCA adherence. Notes: $P$-value was obtained using the $t$-distribution test.

Abbreviations: DN, diabetic neuropathy; SDSCA, Summary of Diabetes Self-Care Activities; MNSI, Michigan Neuropathy Screening Instrument. relevant improvements in their HbA1c levels. DN is directly linked to a prolonged diabetic status and a poor glycemic control. ${ }^{16}$ Furthermore, the results of the sensory and motor Nerve Conduction Sensitivity (NCS) tests have been found to be significantly influenced by an abnormal glycemic hemoglobin level and by the hyperglycemia's severity. ${ }^{17}$

This analysis showed that the presence of both neuropathy and depression lowers SDSCA levels in diabetic patients. Poor self-care leads to poor glycemic control, development, and/or aggravation of neuropathy, leading further to the onset and/or aggravation of depression and thus creating a continuous loop-type relationship among the components, with major negative implications for the patient's health status. It is of paramount importance that this loop be interrupted by improving the overall medical care through the enrichment of the therapeutic strategy for the patient's diabetes, emphasizing the importance of self-care, adhering to diet and exercise, preventing or treating neuropathy, and offering psychological counseling for avoiding the onset of depression or treating it once present.

Depression and emotional distress have often been suggested to influence self-care behaviors in a negative manner, implicitly influencing glycemic control, ${ }^{18,19}$ concentrating studies on negative emotional conditions. A significant number of these studies found correlations between negative emotional conditions and both reduced self-care activities ${ }^{20,21}$ and an elevation in $\mathrm{HbA} 1 \mathrm{c}$ values. ${ }^{22-26}$ This is the first study of its kind on the Romanian population, and its results confirm previous discoveries in the field.

This study's findings are supported by others that have confirmed the correlation between DN, depression, and selfcare behaviors in the sense that in patients who suffered from severe neuropathy, a very high depression rate was found. ${ }^{9}$ 
Table 3 Correlations between DN severity and SDSCA adherence in patients with T2DM

\begin{tabular}{llll}
\hline SDSCA component & Global MNSI score & MNSI - questionnaire & MNSI - clinical \\
\hline Global score & $-0.527^{*}$ & $-0.392^{*}$ & $-0.440^{*}$ \\
Diet score & $-0.507^{*}$ & $-0.389^{*}$ & $-0.365^{*}$ \\
Exercise score & $-0.380^{*}$ & $-0.314^{*}$ & $-0.267^{*}$ \\
Glycemic monitoring score & $-0.275^{*}$ & $-0.243^{*}$ & $-0.233^{*}$ \\
Foot care score & $-0.238^{*}$ & -0.080 & $-0.33 I^{*}$ \\
PHQ-9 score & $0.495^{*}$ & $0.520^{*}$ & $0.225^{*}$ \\
\hline
\end{tabular}

Notes: *Correlations are significant at $\alpha<0.0$ I threshold. P-value was obtained using the $t$-distribution test.

Abbreviations: DN, diabetic neuropathy; SDSCA, Summary of Diabetes Self-Care Activities; T2DM, type 2 diabetes mellitus; MNSI, Michigan Neuropathy Screening Instrument; PHQ-9, Patient Health Questionnaire-9.

These components have also been investigated in young adults, revealing that they might need more psychological and self-care assistance, given their higher depression scores and the fact that they were more likely to have depressive symptoms that bore clinical significance. ${ }^{27}$ Another study showed that nonadherence to diabetic self-care activities is present even when low levels of depressive symptomatology are found and that significantly better self-care can be achieved by alleviating the latter. ${ }^{11}$ The main strength of this study is that it is the only study of its kind performed on the Romanian population, having gathered data from a total of 198 patients. Data regarding the impact of DN on self-care are scarce, even though self-care is vital in the management of diabetes and it is important that we focus on it when elaborating a strategy for the patient. The results of this study may have multiple impacts on the health care policies and practices in Romania regarding the management of patients with multiple chronic diseases (including T2DM here): in these patients, a series of self-management education programs should be initiated in parallel to an increased proactive screening for T2DM chronic complications, including DN and depressive disorder. Moreover, in patients with DN, special care and education programs should be implemented aiming to enhance, or at least not to deteriorate, the quality of DRSCA. The limitation of this study is represented by the fact that it is a cross-sectional, noninterventional study, while the complications of diabetes occur over time. Furthermore, it investigates the present self-care activities of the patient and does not render any information about the patient's past, which is of importance due to the fact that the neuropathy debuted in a past about which we have no information regarding self-care.

As further perspectives, we intend to follow-up with the patients enrolled, assessing the progress of the neuropathy in relation to their attitude toward self-care activities and also evaluating the progress of SDSCA when treating/improving the neuropathy/depression; that is, if patients who find themselves in a better state will show an increased adherence.

\section{Conclusion}

The presence of DN is associated with decreases in the quality of DRSCA's adherence in patients with DM and with increases in depression's symptomatology. The significant, negative association between the severity of DN and the quality of disease self-management points to a possible loop-type relationship between these two components, being possible a reciprocal augmentation with negative consequences on the global management of the disease. In order to avoid a decrease in the quality of disease self-management, special self-management-related education should be provided in insulin-treated patients with DN.

\section{Acknowledgments}

This research was supported by an internal grant, funded by "Victor Babes" University of Medicine and Pharmacy, Timisoara, Romania (PLURINEURODIAB. PII-C4-TC2016-16441-01).

\section{Disclosure}

The authors report no conflicts of interest in this work.

\section{References}

1. International Diabetes Federation. IDF Diabetes Atlas. 7th ed. Brussels, Belgium: International Diabetes Federation; 2015.

2. Centers for Disease Control and Prevention. National Diabetes Statistics Report: Estimates of Diabetes and Its Burden in the United States, 2014. Atlanta, GA: US Department of Health and Human Services; 2014.

3. Centers for Disease Control and Prevention. National Diabetes Fact Sheet, 2011. Atlanta, GA: Centers for Disease Control and Prevention, US Dept. of Health and Human Services; 2011.

4. Callaghan BC, Price RS, Feldman EL. Distal symmetric polyneuropathy: a review. JAMA. 2015;314(20):2172-2181.

5. Papanas N, Ziegler D. Risk factors and comorbidities in diabetic neuropathy: an update 2015. Rev Diabet Stud. 2015;12(1-2):48-62.

6. Botelho MC, Conde MG, Rebelo Braz NM. Functional aspects in ageing adults with diabetic neuropathy. A review. Curr Diabetes Rev. 2015; 12(2):114-119.

7. Karmakar C, Jelinek HF, Khandoker A, et al. Multi-lag HRV analysis discriminates disease progression of post-infarct people with no diabetes versus diabetes. In: Proceedings at the 37th Annual International Conference of the IEEE, Engineering in Medicine and Biology Society (EMBC); August 25-29, 2015; Milan, Italy. 
8. Balcığlu AS, Müderrisoğlu H. Diabetes and cardiac autonomic neuropathy: clinical manifestations, cardiovascular consequences, diagnosis and treatment. World J Diabetes. 2015;6(1):80-91.

9. Rekleiti M, Sarafis P, Saridi M, et al. Investigation of depression in Greek patients with diabetic peripheral neuropathy. Glob J Health Sci. 2013;5(5):107-114.

10. Hudson JL, Bundy C, Coventry PA, Dickens C. Exploring the relationship between cognitive illness representations and poor emotional health and their combined association with diabetes self-care. A systematic review with meta-analysis. J Psychosom Res. 2014;76(4):265-274.

11. Gonzalez JS, Safren SA, Cagliero E, et al. Depression, self-care, and medication adherence in type 2 diabetes: relationships across the full range of symptom severity. Diabetes Care. 2007;30(9):2222-2227.

12. Herman WH, Pop-Busui R, Braffett BH, et al. Use of the Michigan Neuropathy Screening Instrument as a measure of distal symmetrical peripheral neuropathy in Type 1 diabetes: results from the Diabetes Control and Complications Trial/Epidemiology of Diabetes Interventions and Complications. Diabet Med. 2012;29(7):937-944.

13. Toobert D, Hampson S, Glasgow RE. The summary of diabetes self-care activities measure: results from 7 studies and a revised scale. Diabetes Care. 2000;23(7):943-950.

14. Spitzer RL, Kroenke K, Williams JB. Patient Health Questionnaire Study Group. Validity and utility of a self-report version of PRIME-MD: the PHQ Primary Care Study. JAMA. 1999;282(18):1737-1744.

15. Schmitt A, Gahr A, Hermanns N, Kulzer B, Huber J, Haak T. The Diabetes Self-Management Questionnaire (DSMQ): development and evaluation of an instrument to assess diabetes self-care activities associated with glycemic control. Health Qual Life Outcomes. 2013;11:138.

16. Nisar MU, Asad A, Waqas A, et al. Association of diabetic neuropathy with duration of type 2 diabetes and glycemic control. Cureus. 2015; 7(8):e302.

17. Hsu HY, Chiu HY, Lin HT, Su FC, Lu CH, Kuo LC. Impacts of elevated glycemic hemoglobin and disease duration on the sensory-motor control of hands in diabetes patients. Diabetes Metab Res Rev. 2015 31(4):385-394.

18. Piette JD, Richardson C, Valenstein M. Addressing the needs of patients with multiple chronic illnesses: the case of diabetes and depression. $\mathrm{Am}$ J Manag Care. 2004;10(2 Pt 2):152-162.
19. Peyrot M, McMurry JF Jr, Kruger DF. A biopsychosocial model of glycemic control in diabetes: stress, coping and regimen adherence. J Health Soc Behav. 1999;40(2):141-158.

20. Gonzalez JS, Peyrot M, McCarl LA, et al. Depression and diabetes treatment nonadherence: a meta-analysis. Diabetes Care. 2008;31(12): 2398-2403.

21. Gonzalez JS, Delahanty LM, Safren SA, Meigs JB, Grant RW. Differentiating symptoms of depression from diabetes-specific distress: relationships with self-care in type 2 diabetes. Diabetologia. 2008;51(10): 1822-1825.

22. Aikens JE, Perkins DW, Lipton B, Piette JD. Longitudinal analysis of depressive symptoms and glycemic control in type 2 diabetes. Diabetes Care. 2009;32(7):1177-1181.

23. Fisher L, Glasgow RE, Strycker LA. The relationship between diabetes distress and clinical depression with glycemic control among patients with type 2 diabetes. Diabetes Care. 2010;33(5):1034-1036.

24. Fisher L, Mullan JT, Arean P, Glasgow RE, Hessler D, Masharani U. Diabetes distress but not clinical depression or depressive symptoms is associated with glycemic control in both cross-sectional and longitudinal analyses. Diabetes Care. 2010;33(1):23-28.

25. Lustman PJ, Anderson RJ, Freedland KE, de Groot M, Carney RM, Clouse RE. Depression and poor glycemic control: a meta-analytic review of the literature. Diabetes Care. 2000;23(7):934-942.

26. Pibernik-Okanovic M, Grgurevic M, Begic D, Szabo S, Metelko Z. Interaction of depressive symptoms and diabetes-related distress with glycaemic control in type 2 diabetic patients. Diabet Med. 2008;25(10): 1252-1254.

27. Browne JL, Nefs G, Pouwer F, Speight J. Depression, anxiety and self-care behaviours of young adults with type 2 diabetes: results from the International Diabetes Management and Impact for Longterm Empowerment and Success (MILES) Study. Diabet Med. 2015; 32(1):133-140.
Patient Preference and Adherence

\section{Publish your work in this journal}

Patient Preference and Adherence is an international, peer-reviewed, open access journal that focuses on the growing importance of patient preference and adherence throughout the therapeutic continuum. Patient satisfaction, acceptability, quality of life, compliance, persistence and their role in developing new therapeutic modalities and compounds to optimize

\section{Dovepress}

clinical outcomes for existing disease states are major areas of interest for the journal. This journal has been accepted for indexing on PubMed Central. The manuscript management system is completely online and includes a very quick and fair peer-review system, which is all easy to use. Visit http://www. dovepress.com/testimonials.php to read real quotes from published authors. 